\title{
Targeting Insulin-Like Growth Factor-1 Signaling into the Central Nervous System for Promoting Myelin Repair
}

\author{
Nadine Wilczak, Jacques De Keyser and Daniel Chesik \\ Department of Neurology, University Medical Center Groningen, the Netherlands.
}

\begin{abstract}
Multiple sclerosis (MS) is the most common demyelinating disease of the central nervous system (CNS). Without myelin, nerve impulses in the CNS are slowed or stopped, leading to a constellation of neurological symptoms. Demyelination also provides a permitting condition for irreversible axonal damage. Remyelination of MS lesions largely fails, although oligodendrocyte precursors and premyelinating oligodendrocytes (myelin forming cells) are present in many demyelinated plaques. Insulin-like growth factor (IGF)-1 is a growth factor that should provide the appropriate signals to promote repair of MS lesions, because it acts as a survival factor for cells of the oligodendrocyte lineage and stimulates myelin synthesis. In a pilot study on MS patients, no detectable remyelinating effects in the CNS were observed following subcutaneous administration of IGF-1. A number of reasons might explain a lack of beneficial effects: a) it is unlikely that subcutaneous administration of IGF-1 provides sufficient passage across the blood-brain-barrier and into the CNS, b) the biological actions of IGF-1 are tightly regulated by several insulin-like growth factor binding proteins (IGFBPs), which become upregulated in the demyelinated lesions and may prevent access of IGF-1 to its receptor, c) IGF-1 not only acts on oligodendrocytes, but also stimulates the proliferation of astrocytes, which form the glial scar that impedes repair processes. In this review, we will discuss strategies to enhance IGF-1 signaling in the CNS utilizing a) alternative routes of administration, b) IGF analogues that displace IGF-1 from regulatory IGFBPs and c) strategies to selectively target IGF-1 to oligodendrocytes.
\end{abstract}

Keywords: Insulin-like growth factor(IGF)-1, IGF-1 analogues, IGF-binding proteins, IGFBP ligand inhibitors, microRNAs, multiple sclerosis

\section{Introduction}

Multiple sclerosis (MS) is a chronic multifocal demyelinating disease of the central nervous system (CNS) of unknown etiology. One in thousand persons in Europe are afflicted with this disease which generally occurs between the ages of 30-40 (Noseworthy et al. 2000). Pathological hallmarks of MS are inflammation, demyelination, degeneration and loss of oligodendrocytes, proliferation of astrocytes, and axonal damage in the CNS (Wolswijk, 2000). Figure 1 shows a chronic MS lesion, characterized by demyelination and astrogliosis. The underlying cause of myelin destruction and death of oligodendrocytes in MS is not completely understood, however data suggest that MS may be a disease of autoimmune nature (Steinman, 1996). Disruption of the myelin sheaths, axonal injury and glial scar formation is responsible for the clinical symptoms that accumulate in the course of disease. For a disease with such a high impact, the present therapeutic options are disappointing with restricted clinical benefits.

In order to prevent further damage from occurring in the CNS, current therapies target the immune system and are aimed at reducing relapses, which are caused by the formation of new demyelinating lesions. Current immunomodulatory therapies, including interferon- $\gamma($ Fillipini et al. 2003) and glatiramer acetate, show only a modest protective effect against relapses. Therapies directed at repairing damage, e.g. based on remyelination strategies, have not yet been developed.

Following demyelination, some remyelination of MS lesions can be observed during the early stages of the disease (Prineas et al. 1993), although this is often limited in its extent, and largely fails as the disease progresses. Most chronic lesions of MS demonstrate no remyelination, although oligodendrocyte precursor cells and premyelinating oligodendrocytes are present in many demyelinated plaques (Chang et al. 2002; Scolding et al. 1998; Wolswijk, 2002). This suggests that the microenvironment in chronic MS lesions may possess the potential for remyelination, yet failure of this event is likely due to a lack of appropriate signals to stimulate remyelination. Reduced (neuro) trophic support to

Correspondence: Dr. N. Wilczak, University Medical Center Groningen, Department Neurology, Postbus 30001, Hanzeplein 1, 9700 RB Groningen, The Netherlands. Tel: 0031-50-3637719; Fax: 0031-50-3611707.

Copyright in this article, its metadata, and any supplementary data is held by its author or authors. It is published under the Creative Commons Attribution By licence. For further information go to: http://creativecommons.org/licenses/by/3.0/. 


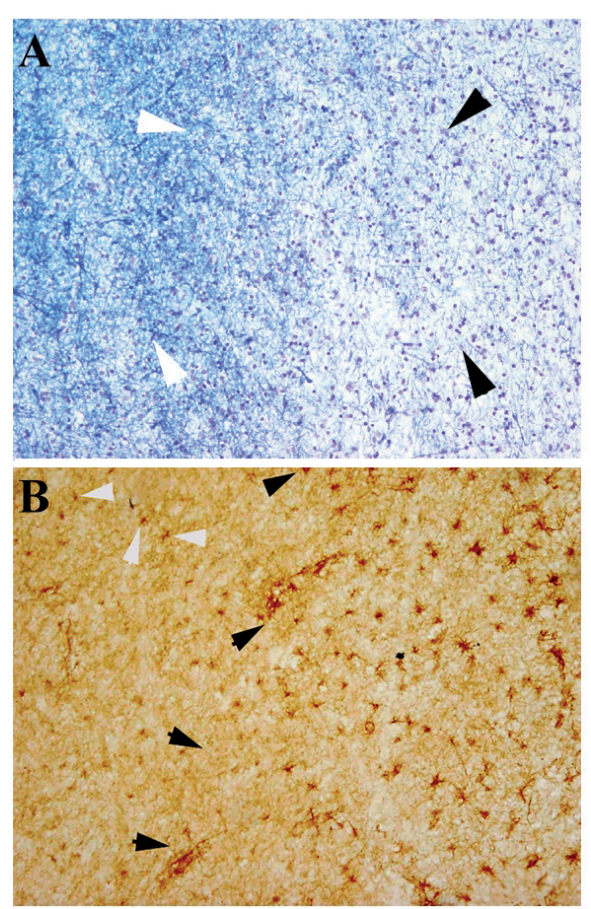

Figure 1. Luxol-fast blue staining (myelin staining) of the white matter from MS (A). The pale area is a demyelinated plaque (black arrows) and the surrounding non-affected area is myelinated white matter (white arrows). GFAP-staining of astrocytes in a MS plaque (B) showing the presence of large reactive astrocytes in MS (black arrows) and normal astrocytes surrounding the MS plaque (white arrows). Astrocytes in the MS plaques are stained using an antibody against glial fibrillary acidic protein (GFAP) a specific marker for astrocytes.

oligodendrocytes might be implicated in oligodendrocyte apoptosis and the failure of remyelination in MS. Insulin-like growth factor (IGF)-1 is a (neuro)trophic growth factor with insulin-like metabolic activities which possesses potential clinical applications, particularly in (neuro) degenerative disorders of the CNS.

\section{Short Overview of the IGF System}

The components of the IGF system include IGF-1 and -2 (IGFs), type-1 and -2 IGF receptors and six insulin-like growth factor binding proteins (IGFBPs) . In the CNS, both IGF-1 and -2 are produced as paracrine and autocrine hormones. IGF-2 is genetically related to IGF-1 and both hormones display approximately 62 percent sequence homology. Whereas IGF-1 expression in the CNS is high in neuronal rich regions of the brain (Bondy et al. 1992), IGF-2 is highly expressed in mesenchymal support structures of the CNS, including the choroid plexus (Logan et al. 1994).
Biological actions of these growth factors on target cells are mediated by cell-surface receptors. Two types of IGF receptors have been identified in human brain, the type-1 and type-2 IGF receptor. The type-1 IGF receptor is a membrane glycoprotein consisting of two $\alpha$-subunits and two $\beta$-subunits (Yamasaki et al. 1993). The $\alpha$-subunit is entirely extracellular and contains the ligandbinding site. The $\beta$-subunit contains a transmembrane domain with a short extracellular region, and a tyrosine kinase domain in its cytoplasmic portion. The type-2 IGF receptor is structurally and functionally quite different from the type-1 IGF receptor. The type-2 IGF receptor consists of a single glycosylated polypeptide. The type-2 IGF receptor lies primarily extracellular with a short cytoplasmic tail and consists of 15 repeated mannose6-phosphate (M6P)-binding units (Kornfeld et al. 1992). The major functions of the type-2 IGF receptor binding appear to be lysosomal enzyme trafficking (Kornfeld et al. 1992) and IGF-2 degradation via receptor mediated-internalization (Morgan et al. 1987). Type-2 IGF receptors are not thought to be involved in cell signaling. There is ample consensus today that the biological actions of both IGF-1 and -2 are mediated through type-1 IGF receptors.

In vitro and in vivo, IGF-1 stimulates DNA synthesis and cell growth. A cellular action of IGF1 that is complementary to its stimulation of cell proliferation is its capacity in certain cells to inhibit apoptosis. IGF-1 also induces differentiation of neurons and oligodendrocytes (Feldman et al. 1997). Two major pathways induce the actions of IGF-1 and IGF-2 through type-1 IGF receptors, the first is the mitogen-activated protein (MAP) kinase pathway and the second is the serine-threonine protein kinase Akt pathway. The MAP kinase pathway is often associated in proliferation and differentiation (Kim et al. 1997), whereas the Akt pathway is induced in IGF-1 mediated cell survival (Brunet et al. 1999) and protection from apoptosis (Kermer et al. 2000).

Biological actions of IGF-1 and IGF-2 are modulated through six IGFBPs. These proteins contain high affinity binding sites for IGF-1 and IGF-2, and they possess an 80\% sequence homology with each other (Rajaram et al. 1997). In the circulation, the major IGFBP form is IGFBP-3, which binds IGF-1 and IGF-2 with similar affinities (Baxter, 1994). Circulating IGFBP-3 inhibits insulin-like activity, regulates the rate of transport 
and prolongs half-lives of plasma IGF-1 and IGF-2. As carrier proteins of IGF-1 and IGF-2, a major function of IGFBPs is to transport and target IGFs to specific tissues and cell types. Therefore, these proteins have a central position in IGF ligand-receptor interactions by influencing bioavailability and extracellular distribution. IGFBPs are able to enhance or inhibit IGF effects by regulating binding to type-1 IGF receptors, a mechanism that is thought to be dependent on specific cell and tissue properties (Firth and Baxter, 2002). Binding of IGF/IGFBP complexes to components of the extracellular matrix (ECM) and the cell-surface can facilitate the release of IGFs leading to enhanced delivery to type- 1 and type-2 IGF receptors. For example, the binding of IGFBP-2 to chondroitin6-sulphate, an ECM component, decreases the binding affinity of IGFBP-2 to IGF-1 by 3 -fold in rat brain, leading to increased levels of biologically active IGF-1 (Russo et al. 1997). Another mechanism involved in IGF regulation are specific proteases, which are secreted by cells and act as growth stimulators by increasing local IGF availability (Conover et al. 1993). Circulating and tissue specific IGFBPs bind IGF-1 and IGF-2 by forming biologically inactive IGF/IGFBP complexes. IGF-1 and -2 can be released from these complexes, for example by proteolysis of IGFBPs. IGFBP fragments generated by the action of cellular proteases show a marked loss of IGF binding affinity.

In conclusion, IGFBPs can influence IGF actions in different ways. The multitude of these effects depends on the cell type in which they are expressed, how they interact with cell surfaces and ECM, the presence of specific proteases and the formation of high affinity binding proteins complexed with IGFs.

\section{The Role of Insulin-Like Growth Factor-1 in Multiple Sclerosis}

During nervous system development, IGF-1 plays a crucial role in cell proliferation, differentiation, and survival (Bondy and Cheng, 2004; Russo et al. 2005). During that period IGF-1 and type-1 IGF receptors are highly expressed in neuronal rich regions, such as the spinal cord, midbrain, cerebral cortex, hippocampus, and olfactory bulb (Anlar et al. 1999; Beck et al. 1988). We have shown that cerebral white matter in human neonates, undergoing active myelination, contains a 3-fold higher density of type-1 IGF receptors than in adults, indicating that IGF-1 also plays a important role in the myelination of the human CNS (De Keyser et al. 1994b).

In vitro experiments have demonstrated that IGF-1 greatly enhances oligodendrocyte survival (McMorris et al. 1986), myelin production (Roth et al. 1995) and proliferation of oligodendrocyte precursors (Mozell and McMorris, 1991). The effects of IGF-1 are not only restricted to oligodendrocytes of the CNS, but also apply to Schwann cells of the pheripheral nervous system, which demonstrate enhanced differentiation, myelin production, and increased survival in response to IGF-1 stimulation (Cheng et al. 1999; Ogata et al. 2004; Syroid et al. 1999).

The importance of IGF-1 in myelin production has also been demonstrated in several animal models. In the cuprizone model, demyelination occurs in the corpus callossum and superior cerebellar peduncles, and remyelination in these areas ensues when treatment with cuprizone is terminated. Cuprizone-induced demyelination in mice deficient of type-1 IGF receptors has demonstrated inadequate remyelination and lack of oligodendrocyte progenitor cells (OPC) accummulation in the site of injury (Mason et al. 2003). Transgenic mice that overexpress IGF-1 show increased brain growth and myelination (Carson et al. 1993). Myelin content in these animals was increased by $130 \%$. By comparison, IGF-1 knock-out mice displayed reduced brain size, hypomyelination, reduced density of oligodendrocytes, loss of neuron populations, as well as reduced glucose uptake (Beck et al. 1995; Cheng et al. 2000; Liu et al. 1993). Transgenic mice overexpressing IGFBP-1, an inhibitory binding protein for IGF-1, showed reduced myelinated axons and thickness of the myelin sheets, this feature was induced by reduction in myelin expression (Ye et al. 1995). Other studies have shown that systemic application of IGF-1 in acute demyelinating experimental autoimmune encephalomyelitis (EAE), an animal model for MS, significantly reduced the number and area of the demyelinated lesions in the spinal cord (Liu and Yao, 1995; Yao et al. 1995). IGF-1 increased the number of remyelinated axons, reduced the permeability of the blood-spinal cord barrier, and enhanced myelin expression.

However, in chronic-relapsing EAE, systemic administration of IGF-1 together with IGFBP-3 after disease onset resulted in increased severity and or relapses (Lovett-Racke et al. 1998). In line 
with these results, long-term application of IGF-1 in chronic- relapsing EAE from the standpoint of myelin gene expression and repair, showed no positive effects of IGF-1 (Canella et al. 2000). In a clinical trial with systemically administered recombinant IGF-1 in MS patients, no effects on either new lesion formation or remyelination of existing lesions could be demonstrated (Frank et al. 2002). Data from this clinical trial, as well as from the above mentioned study on chronic relapsing EAE raise doubts about the effectiveness of systemic administered IGF-1 in chronic forms of demyelination. In the following sections, we dicuss several obstacles that might explain a lack of beneficial effects of systemic administered IGF-1.

\section{Enhancing the Level of Free IGF-1 and Targeting IGF-1 into the CNS}

One possible obstacle in an IGF-1 based therapy is the limited penetration across the blood brain barrier $(\mathrm{BBB})$ that prevents free passage of large proteins into the CNS. However, there is some evidence that IGF-1 from the circulation might be transported across these barriers, albeit to a limited extent. One possible mechanism to transport IGF-1 from the circulation into the CNS is throughreceptor mediated transcytosis (Reinhardt and Bondy, 1994). However, it is still not clear which receptor is involved in such transport system. It has been suggested that the presence of type-1 IGF receptors on the endothelium might play a role in such transport. The transport of peripheral IGF-1 into the CNS may also be influenced by IGFBPs which become saturable at the BBB (Weihong and Kastin, 2000). Other studies have shown that the uptake of circulating IGFs into cerebrospinal fluid (CSF) and probably into the CNS appears to be independent of type-1 IGF receptors as well as IGF-binding proteins. There is evidence that choroid plexus megalin is involved in neuroprotection by serum IGF-1 in Alzheimer disease by its dual effects on transporting IGF-1 in across the BBB, and by enhancing the clearance of brain amyloid$\beta$ (Carro et al. 2005). However, it remains questionable whether systemic levels of IGF-1 would indeed raise ligand levels in the deep white matter of the CNS in which demyelination occurs. The amount of IGF-1 eventually reaching the deep white matter will depend on the concentration of circulating IGF-1 and how avidly the BBB transport system uptakes it.
Approaches that bypass the BBB and show that IGF-1 has neuroprotective effects in the CNS of animals use surgically invasive procedures, such as the intracerebrovascular or intraparenchymal administration. These methods are not applicable to MS patients but do demonstrate a need for targeting. Intranasal administration of IGF-1 offers an alternative strategy to bypass the BBB and is a method that provides several advantages; it is a non-invasive way of drug application, there is a direct delivery of the drug into the CNS, levels of the drugs are more concentrated by avoiding the diluting effects of the circulation, and degradation and destruction of drugs are minimized by avoiding the gastrointestinal tracts. Thorne and co-workers have demonstrated that intranasal application of IGF-1 results in a rapid delivery of IGF-1 into multiple areas of the CNS, including the deep white matter in which demyelination can occur (Thorne et al. 2004). In this study, IGF-1 was delivered from the nasal cavity along olfactory and trigeminal pathways and was accompanied by activation of signaling pathways in several areas that express high levels of type-1 IGF receptors. Thorne and coworkers suggested an extracellular route of transportation from the nasal passages into the CNS associated with components of the trigeminal nerve. This mode of transport provides evidence for a rapid and direct pathway for protein transport into the CNS following intranasal administration.

A second obstacle in an IGF-1 based therapy lies in the complex regulation of IGF-actions. The biological effects of IGF-1 on target cells are mediated by interactions with the type-1 IGF receptor (LeRoith et al. 1995). However, six IGFBPs govern this interaction (Clemmons, 1997; Spagnoli and Rosenfeld, 1997). All six IGFBPs may inhibit IGF-1 actions by sequestering IGF-1, thereby preventing the interaction of IGF-1 to its receptor. We have found that IGFBP-1 and IGFBP-6 were upregulated on oligodendrocytes in the periplaque white matter in MS lesions (unpublished results). Furthermore, it has been demonstrated that these IGFBPs bind IGF-1 with high affinity, and inhibit IGF-1 induced survival and myelin production of primary oligodendrocytes (Kühl et al. 2002, 2003). These results suggest that the up-regulation of IGFBPs lead to a shortage of biologically active (free) IGF-1 in the demyelinated MS plaques, contributing to the lack of (re)-myelination. However, the exact function of IGFBP-1 and IGFBP-6 
in physiological and pathological situations is yet unknown.

IGF-1 analogues that display low affinity for IGFBPs, and IGF-1 analogues that display high affinity for IGFBPs, which are able to displace endogenous IGF-1 and or IGF-2 from IGFBPs, may be suitable candidates for stimulating (re) myelination in MS (Fig. 2). Des(1-3) IGF-1 is a truncated form of IGF-1 lacking the tripeptide glycine-proline-glutamate (GPE) at the aminoterminus of the full-length form (Sara et al. 1986). The biological potency of this analogue is significantly higher than that of the full-length form and is explained by its reduced affinity for IGFBPs. IGF-1 analogues that display high affinity for IGFBPs, which can therefore displace endogenous

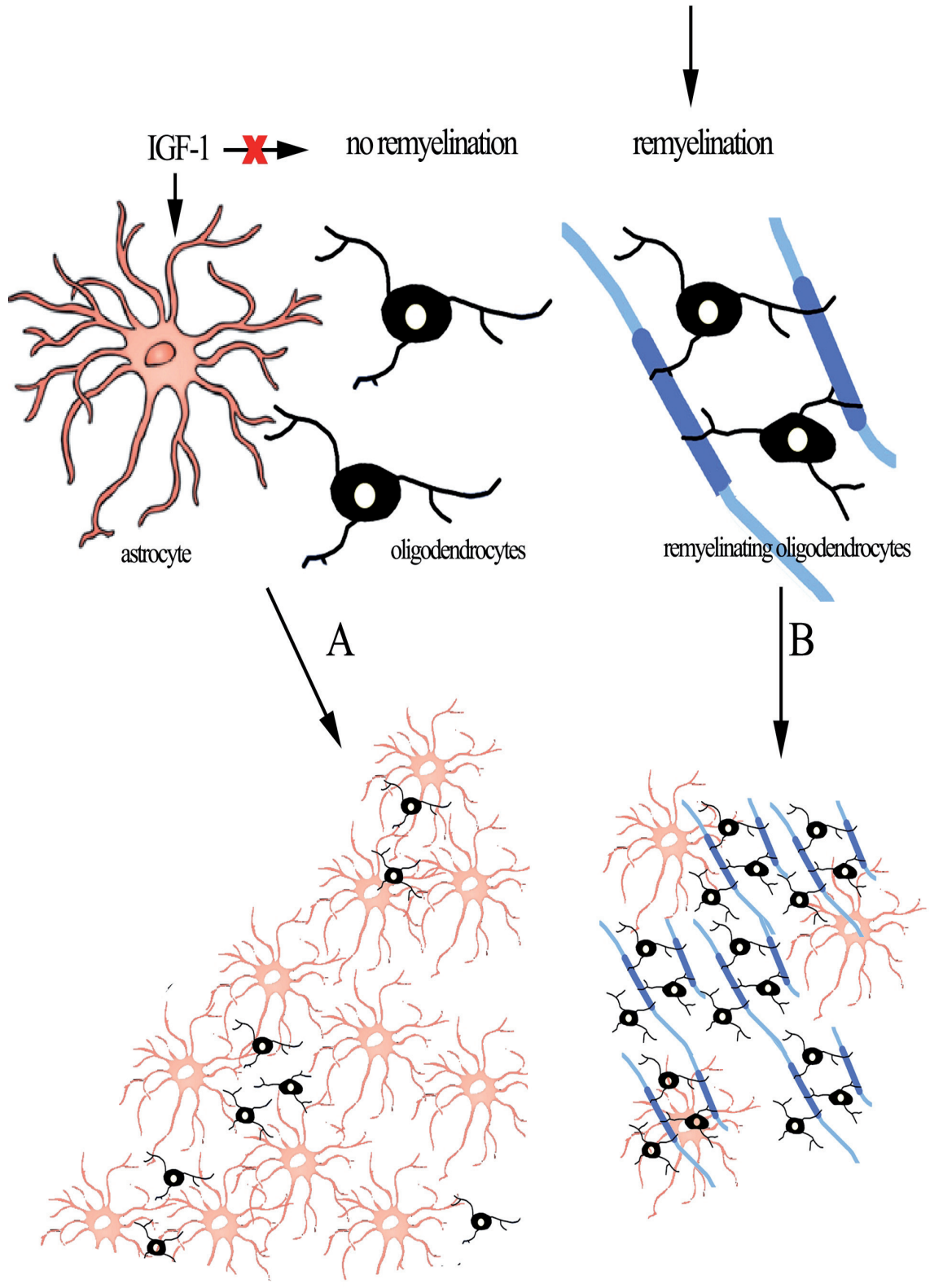

IGF-1 analogues

IGFBP ligand inhibitors

Figure 2. A schematic overview: Astrocytes regulate their own cellular action through IGF-1, and Astrocytic IGF-1 has no effect on (pre)myelinating oligodendrocytes in MS. Astrocytes become astrogliotic forming the tissue scar in MS lesions and preventing remyelination by oligodendrocytes (A). Through intranasal application of IGF-1 analogues and or IGFBP ligand inhibitors, IGF-1 is released from IGFBPs which are present on oligodendrocytes and IGF-1 become available to oligodendrocytes, resulting in remyelination (B). 
IGF-1 from IGFBPs might also be considered. This approach has already been studied in rats with focal cerebral ischemia. In this model, the intracerebroventricular administration of the IGF-1 analogue [( $\left.\mathrm{Leu}^{24,59,60}, \mathrm{Ala}^{31}\right)$ hIGF-1] with high affinity to IGFBPs and no affinity to type-1 IGF receptors, increased levels of biologically active IGF-1 and provided potent neuroprotection (Loddick et al. 1998).

A third obstacle lies in the targeting of IGF-1 signaling to the myelin producing cells: the oligodendrocyte. Such a targeting is complicated by the high expression of type-1 IGF receptors on other cells in the CNS. We have demonstrated that type-1 IGF receptors were present on neurons and other glial cells, including microglia and astrocytes (De Keyser et al. 1994a; Wilczak and De Keyser, 1997). This may have important implications for the clinical use of IGF-1 in MS. Chronic plaques of MS contain a dense network of astrocytes, which are responsible for the characteristic astrogliotic plaque. In vitro studies have shown that IGF-1 enhances the proliferation of astrocytes (Chesik et al. 2004; Tranque et al. 1992). As acute MS lesions are rapidly invaded by reactive astrocytes, enhancing the levels of IGF-1 in MS lesions may not only protect oligodendrocytes and stimulate remyelination but also enhance the astrogliosis that creates a glial scar, limiting remyelinating processes. For these reasons, targeting IGF-1 effects to oligodendrocytes is crucial. A feasible approach for targeting oligodendrocytes consists of intervening in IGF-1 regulation by IGFBPs. In MS lesions, we have shown a unique distribution of IGFBPs expression on oligodendrocytes and astrocytes. Oligodendrocytes in demyelinated MS plaques display an upregulation of IGFBP-1 and IGFBP-6, whereas astrocytes show increased expression of IGFBP-2 and IGFBP-4 (Chesik et al. 2006). An interesting prospect is the use of non-peptide small molecules that act as specific IGFBP ligand inhibitors and prevent binding of IGF-1 to specific IGFBPs (Chen et al. 2001; Zhu et al. 2003). This approach would result in an elevation of biological active IGF-1 that is available in the vicinity of olgodendrocytes. NBI-31772 is a recently developed non-peptide small molecule that binds to all six known IGFBPs and displaces biologically active IGF-1 from all six binding proteins (Liu et al. 2001). The neuroprotective effects of NBI31772 has been studied in experimental models of cerebral ischemia. This study has demonstrated that intracerebroventricular administration of NBI31772 significantly reduced ischemic brain damage and infarct size. (Mackay et al. 2003). New potent ligand-inhibitors could be designed which selectively bind individual IGFBPs in order to enhance and target bioactive IGF-1.

Another approach to elevate IGF-1 levels as well as target IGF-1 into oligodendrocytes is altering the expression level of IGFBPs on oligodendrocytes and astrocytes in MS. IGFBP-2 is of particular interest because hypertrophic astrocytes in MS lesions express high levels of IGFBP-2 (Chesik et al. 2006). Astrocytes are the primary source of IGF-1 in damaged CNS, and it has been suggested that this growth factor assists in neuronal protection as well as in facilitation of myelin production. We have shown that reactive astrocytes in vitro and in situ upregulate IGFBP-2 and that combined treatment of IGFBP-2 and IGF-1 does not inhibit IGF-1 stimulated astrocyte proliferation, whereas it inhibits IGF-1 stimulated survival of oligodendrocytes (Chesik et al. 2004). We propose that an upregulation of IGFBP-2 in MS facilitates the process of astrogliosis by targeting IGF-1 to these cells. Inhibition of endogenous expression of IGFBP-2 in astrocytes by means of microRNAs (miRNAs) might have implications on cell proliferation and maturation of these cells. In MS, astrocytes become reactive and form the glial scar, which is thought to impede remyelination processes. MiRNAs are a growing family of singlestranded forms of RNA, which regulate the expression and production of proteins. Such an approach was already investigated in neuroblastoma (NB) cells (Tanno et al. 2005). Neuroblastoma is a malignant childhood tumor, in which IGFBP-5 is frequently expressed. By suppressing the expression level of IGFBP-5 in NB cells through miRNAs, NB cells become more prone to apoptosis (Tanno et al. 2005). The use of these molecules as therapeutics to influence the expression of IGFBPs during demyelination has a long way to go. However, regulating the expressing of IGFBPs through miRNAs opens a new window to selectively study the role of IGFBPs in several diseases such as MS.

\section{Conclusion}

The use of IGF-1 analogues, IGFBP ligand inhibitors and microRNAs to modify actions of IGF-1 signaling and induce remyelination in the CNS 
would be a tremendous breakthrough in the treatment of human demyelinating diseases, such as MS. The unique distribution of IGFBPs in MS lesions as well as a unique functional characteristic of the IGF-1 receptor on oligodendrocytes offers a means to specifically target this cell type.

\section{Acknowledgments}

This work was supported by the School of Behavior and Cognitative Neurosciences (BCN) Groningen and "MSanders", The Netherlands.

\section{References}

Anlar, B., Sullivan, K.A. and Feldman, E.L. 1999. Insulin-like growth factor-I and central nervous system development. Horm. Metab. Res., $31: 120-5$.

Baxter, R.C. 1994. Insulin-like growth factor binding proteins in the human circulation: a review. Horm. Res., 42:140-4.

Beck, F., Samani, N.J., Byrne, S. et al. 1988. Histochemical localization of IGF-1 and IGF-II mRNA in the rat between birth and adulthood. Development, 104:29-39.

Beck, K.D., Powell-Braxton, L., Widmer, H.R. et al. 1995. Igf1 gene disruption results in reduced brain size, CNS hypomyelination, and loss of granule and striatal parvalbumin-containing neurons. Neuron, 14:717-30.

Bondy, C.A. and Cheng, C.M. 2004. Signaling by insulin-like growth factor I in brain. Eur. J. Pharmacol., 490:25-31.

Bondy, C., Werner, H., Roberts, CT., Jr. et al. 1992. Cellular pattern of type-I insulin-like growth factor receptor gene expression during maturation of the rat brain: comparison with insulin-like growth factors I and II. Neuroscience, 46:909-23.

Brunet, A., Bonni, A., Zigmond, M.J. et al. 1999. Akt promotes cell survival by phosphorylating and inhibiting a Forkhead transcription factor. Cell, 96:857-68.

Canella, B., Pitt, D., Capello, E. et al. 2000. Insulin-like growth factor-1 fails to enhance central nervous system myelin repair during autoimmune demyelination. Am. J. Pathol., 157:933-43.

Carson, M.J., Behringer, R.R., Brinster, R.L. et al. 1993. Insulin-like growth factor-I increases brain growth and central nervous system myelination in transgenic mice. Neuron, 10:729-40.

Caro, E., Spuch, C., Trejo, J.L. et al. 2005. Choroid plexus megalin is involved in neuroprotection by serum insulin-like growth factor-I. J. Neurosci., 25:10884-93.

Chang, A., Tourtellotte, W.W., Rudick, R. et al. 2002. Premyelinating oligodendrocytes in chronic lesions of multiple sclerosis. N. Engl. $J$. Med., 346:165-73.

Chen, C., Zhu, Y.F., Liu, X.J. et al. 2001. Discovery of a series of non-peptide small molecules that inhibit the binding of insulin-like growth factor (IGF) to IGF-binding proteins. J. Med. Chem., 44:4001-10.

Cheng, C.M., Reinhardt, R.R., Lee, W.H. et al. 2000. Insulin-like growth factor-I regulates developing brain glucose metabolism. Proc. Natl. Acad. Sci., U.S.A., 97:10236-41.

Cheng, H.L., Russel, J.W., Feldman, E.L. et al. 1999. IGF-I promotes peripheral nervous system myelination. Ann. N.Y. Acad. Sci., 883:124-30.

Chesik, D., De Keyser, J., Glazenburg, L. et al. 2006. Insulin-like growth factor binding proteins: regulation in chronic active plaques in multiple sclerosis and functional analysis on glial cells. Eur. J. Neurosci., 24:1645-52.

Chesik, D., Kühl, N.M., Wilczak, N. et al. 2004. Enhanced production and proteolytic degradation of insulin-like growth factor binding protein2 in proliferating astrocytes. J. Neurosci. Res., 77:354-62.
Clemmons, D.R. 1997. Insulin-like growth factor binding proteins and their role in controlling IGF actions. Cytokine Growth Factor Rev., $8: 45-62$.

Conover, C.A., Kiefer, M.C. and Zapf, J. 1993. Posttransitional regulation of insulin-like growth factor binding protein-4 in normal and transformed human fibroblasts. Insulin-like growth factor dependence and biological study. J. Clin. Endocrinol. Metab., 91:1129-37.

De Keyser, J., Wilczak, N., De Backer, J.P. et al. 1994a. Insulin-like growth factor-I receptors in human brain and pituitary gland: an autoradiographic study. Synapse, 17:196-202.

De Keyser, J., Wilczak, N. and Goossens, A. 1994b. Insulin-like growth factor-I receptor densities in human frontal cortex and white matter during aging, in Alzheimer's De disease, and in Huntington's disease. Neurosci. Lett., 172:93-6.

Feldman, E.L., Sullivan, K.A., Kim, B. et al. 1997. Insulin-like growth factors regulate neuronal differentiation and survival. Neurobiol. Dis., 4:201-14.

Fillipini, G., Munari, L., Incorvaia, B. et al. 2003. Interferon's in relapsing remitting multiple sclerose: a systematic review. Lancet, 361:545-52.

Firth, S.M. and Baxter, R.C. 2002. Cellular actions of the insulin-like growth factor binding proteins. Endocr. Rev., 23:824-54.

Frank, J.A., Richert, N., Lewis, B. et al. 2002. A pilot study of recombinant insulin-like growth factor-1 in seven multiple sclerosis patients. Mult. Scler, 8:24-9.

Kermer, P., Klocker, N., Labes, M. et al. 2000. Insulin-like growth factor-I protects axotomised rat retinal ganglion cells from secondary death via PI3-K-dependent Akt phosphorylation and inhibition of caspase3 in vivo. J. Neurosci., 20:2-8.

Kim, B., Leventhal, P.S., Saltiel, A.R. et al. 1997. Insulin-like growth factor-I mediated neurite outgrowth in vitro requires mitogen-activated protein kinase activation. J. Biol. Chem., 272:21268-73.

Kornfeld, S. 1992. Structure and function of the mannose-6-phosphate/ Insulin-like growth factor-II receptors. Ann. Rev. Biochem., 61:307-30.

Kühl, N.M., De Keyser, J., De Vries, H. et al. 2002. Insulin-like growth factor binding protein- 1 and -2 differentially inhibit rat oligodendrocyte precursor survival and differentiation in vitro. J. Neurosci., 69:207-16.

Kühl, N.M., Hoekstra, D., De Vries, H. et al. 2003. Insulin-like growth factor binding protein-6 inhibits survival and differentiation of rat oilgodendrocyte precursor cells. Glia, 44:91-101.

LeRoith, D., Werner, H., Beitner-Johnson, D. et al. 1995. Molecular and cellular aspects of the insulin-like growth factor I receptor. Endocr. Rev., 16:143-63.

Liu, J.P., Baker, J., Perkins, A.S. et al. 1993. Mice carrying null mutations of the genes encoding insulin-like growth factor I (Igf-1) and type 1 IGF receptor (Igf-1r). Cell, 75:59-72.

Liu, X. and Yao, D.L. 1995. Insulin-like growth factor-I treatment reduces clinical deficits and plaques severity in acute demyelinating experimental autoimmune encephalitis. Mult. Scler, 1:2-9.

Liu, X.J., Xie, Q., Zhu, Y.F. et al. 2001. Identification of a nonpeptide ligand that release bioactive insulin-like growth factor-I from its binding protein complex. J. Biol. Chem., 276:32419-22.

Loddick, S.A., Liu, X.J., Lu, Z.X. et al. 1998. Displacement of insulin-like growth factors from their binding proteins as a potential treatment for stroke. Proc. Natl. Acad. Sci., U.S.A., 95:1894-8.

Logan, A., Gonzalez, A.M., Hill, D. et al. 1994. Coordinated pattern of expression and localization of insulin-like growth factor-II (IGF-II) and IGF-binding protein-2 in the adult brain. Endocrinology, 135:2255-64.

Lovett-Racke, A.E., Bittner, P. and Cross, A.H. 1998. Regulation of experimental autoimmune enceplamyelitis with insulin-like growth factor (IGF-I) and IGF-I/IGF-binding protein-3 complex (IGF-I/ IGFBP-3). J. Clin. Invest., 101:1797-804.

Mackay, K.B., Loddick, S.A., Naeve, G.S. et al. 2003. Neuroprotective effects of insulin-like growth factor-binding protein ligand inhibitors in vitro and in vivo. J. Cereb. Blood Flow Metab., 23:1160-7. 
Mason, J.L., Xuan, S. and Dragatsis, I. 2003. Insulin-like growth factor (IGF) signaling through type 1 IGF receptor plays an important role in remyelination. J. Neurosci., 23:7710-8.

McMorris, F.A., Smith, T.M., DeSalvo, S. et al. 1986. Insulin-like growth factor I/somatomedin C: a potent inducer of oligodendrocyte development. Proc. Natl. Acad. Sci., U.S.A., 83:822-6.

Morgan, D.O., Edman, J.C., Standring, D.N. et al. 1987. Insulin-like growth factor II receptor as a multifunctional binding protein. Nature, 329:301-7.

Mozell, R.L. and McMorris, F.A. 1991. Insulin-like growth factor I stimulates oligodendrocyte development and myelination in rat brain aggregate cultures. J. Neurosci. Res., 30:382-90.

Noseworthy, J.H., Luchinetti, C., Rodriguez, M. et al. 2000. Multiple Sclerose. N. Engl. J. Med., 343:938-52.

Ogata, T., Iijima, S., Hoshikawa, S. et al. 2004. Opposing extracellular signal-regulated kinase and Akt pathways controll Schwann cell myelination. J. Neurosci., 24:6724-32.

Prineas, J.W., Barnard, R.O., Kwon, E.E. et al. 1993. Multiple sclerosis: remyelination of nascent plaques. Ann. Neurol., 33:137-51.

Rajaram, S., Baylink, D.J. and Mohan, S. 1997. Insulin-like growth factorbinding proteins in serum and other biological fluids: regulation and function. Endocr. Rev., 18:801-31.

Reinhardt, R.R. and Bondy, C.A. 1994. Insulin-like growth factors cross the blood-brain barrier. Endocrinology, 135:1753-61.

Roth, G.A., Spada, V., Hamill, K. et al. 1995. Insulin-like growth factor I increases myelination and inhibit demyelination in cultured organotypic nerve tissue. Brain Res. Dev. Brain Res., 88:102-8.

Russo, V.C., Bach, L.A., Fosang, A.J. et al. 1997. Insulin-like growth factor binding protein-2 binds to cell surface proteoglycans in the rat brain olfactory bulb. Endocrinology, 138:4858-67.

Russo, V.C., Gluckman, P.D., Feldman, E.L. et al. 2005. The insulin-like growth factor system and its pleiotrophic functions in brain. Endocr. Rev., 26:916-43.

Sara, V.R., Carlsson-Skwirut, C., Anderson, C. et al. 1986. Characterization of somatomedins from human fetal brain: identification of a variant form of insulin-like growth factor-I. Proc. Natl. Acad. Sci., U.S.A., 83:4904-7.

Scolding, N., Franklin, R., Stevens, S. et al. 1998. Oligodendrocyte progenitors are present in the normal adult human CNS and in the lesions of multiple sclerosis. Brain, 121:2221-8.

Spagnoli, A. and Rosenfeld, R.G. 1997. Insulin-like growth factor binding proteins. Curr. Opinion Endocrinol., 4:1-9.
Steinman, L. 1996. Multiple sclerosis: a coordinated immunological attack against myelin in the central nervous system. Cell, 85:299-302.

Syroid, D.E., Zorick, T.S., Arbet-Engels, C. et al. 1999. A role for insulinlike growth factor-I in the regulation of Schwann cell survival. Ageing Res. Rev., 4:195-212.

Tanno, B., Cesi, V., Vitali, R. et al. 2005. Silencing of endogenous IGFBP- 5 by micro RNA interference affects proliferation, apoptosis and differentiation of neuroblastoma cells. Cell. Death Diff., 12:213-23.

Thorne, R.G., Pronk, G.J., Padmanabhan, V. et al. 2004. Delivery of insulinlike growth factor-I to the rat brain and spinal cord along olfactory and trigeminal pathways following intranasal administration. Neuroscience, 127:481-96.

Tranque, P.A., Calle, R., Naftolin, F. et al. 1992. Involvement of protein kinase-C in the mitogenic effects of insulin-like growth factor I on rat astrocytes. Endocrinology, 131:1948-54.

Weihong, P. and Kastin, A.J. 2000. Interactions of IGF-1 with the bloodbrain barrier in vivo and in situ. Neuroendocrinology, 72:171-8.

Wilczak, N. and De Keyser, J. 1997. Insulin-like growth factor-I receptors in normal appearing white matter and chronic plaques in multiple sclerosis. Brain Res., 772:243-6.

Wolswijk, G. 2000. Oligodendrocyte survival, loss and birth in plaques of chronic-stage multiple sclerosis. Brain, 123:105-15.

Wolswijk, G. 2002. Oligodendrocyte precursor cells in demyelinated multiple sclerosis spinal cord. Brain, 125:338-49.

Yamasaki, H., Prager, D. and Melmed, S. 1993. Structure-function of the human insulin-like growth factor receptor-I: a discordance of somatotroph internalization and signaling. Mol. Endocrinol., 7:681-5.

Yao, D.L., Liu, X., Hudson, L.D. et al. 1995. Insulin-like growth factor I treatment reduces demyelination and up-regulates gene expression of myelin-related prpteins in experimental autoimmune encephalomyelitis. Proc. Natl. Acad. Sci., U.S.A., 92:6190-4.

Ye, P., Carson, J. and D' Ercole, A.J. 1995. In vivo actions of insulin-like growth factor-I (IGF-I) on brain myeliation: Studies of IGF-I and IGF binding protein-1 (IGFBP-1) transgenic mice. J. Neurosci., 15:7344-56.

Zhu, Y.F., Wang, X.C., Connors, P. et al. 2003. Quinoline-carboxylic Acids are potent inhibitors that inhibit the binding insulin-like growth factor (IGF) to IGF-binding proteins. Bioorganic. Med. Chem. Lett., 13:1931-4. 